\title{
Toward a comprehensive assessment of functional outcomes in pediatric patients with brain arteriovenous malformations: the Pediatric Quality of Life Inventory
}

\author{
Isaac Josh Abecassis, MD,1 John D. Nerva, MD,1 Jason Barber, MS, ${ }^{1}$ Jason Rockhill, MD, ${ }^{2}$ \\ Richard G. Ellenbogen, MD,' Louis J. Kim, MD,1,3 and Laligam N. Sekhar, MD1,3 \\ Departments of ${ }^{1}$ Neurological Surgery, ${ }^{2}$ Radiation Oncology, and ${ }^{3}$ Radiology, University of Washington, Seattle, Washington
}

OBJECTIVE Brain arteriovenous malformations (bAVMs) are rare in pediatric patients but represent the most common cause of hemorrhagic stroke in this population. Pediatric patients demonstrate superior outcomes in comparison with adult patients with similar lesions and presentations. Most studies of clinical outcomes of pediatric bAVMs use the modified Rankin Scale (mRS), despite a lack of validation in pediatric patients.

METHODS The authors interviewed the parents of 26 pediatric patients who underwent multimodality bAVM treatment and administered the Pediatric Quality of Life Inventory (PedsQL) - a well-validated tool for pediatric outcomes that quantifies performance in a physical, emotional, social, and school domains. They also reviewed clinical information from the patients' medical charts. Statistical analysis was performed using a log-transformed t-test, the Mann-Whitney exact test, the Kruskal-Wallis test, and Spearman correlation. In addition, the literature was reviewed for prior reports of clinical outcome of pediatric cases of bAVM.

RESULTS The average PedSQL health-related quality of life score was $71 \pm 24$, with an average age at diagnosis of 12.5 years and an average follow-up period of 6.8 years. Seventeen patients (65\%) presented with hemorrhage and $4(15 \%)$ with seizures. PedsQL scores correlated strongly and at a statistically significant level $(p<0.001)$ with $\mathrm{mRS}$, Pediatric Overall Performance Category (POPC), Pediatric Cerebral Performance Category (PCPC), and Glasgow Outcome Scale scores. Multivariate modeling validated special education, corrective devices, and cure status as significant predictors of PedsQL scores. Statistically significant risk factors for undergoing placement of a ventriculoperitoneal shunt included lower Glasgow Coma Scale motor scores on admission ( $p=0.042)$, cerebellar location $(p=0.046)$, and nidus volume $(p=0.017)$. Neither treatment modality nor location statistically affected clinical outcomes at follow-up.

CONCLUSIONS There have been few studies of long-term clinical outcomes of bAVM in pediatric patients, and previously published studies have used conventional metrics that have been validated in the adult population, such as the $\mathrm{mRS}$. Although these metrics can serve as reasonable surrogates, an accurate understanding of overall health-related quality of life is contingent on utilizing validated toolsets, such as the PedsQL.

http://thejns.org/doi/abs/10.3171/2016.6.PEDS16103

KEY WORDS pediatric brain arteriovenous malformations; clinical outcomes; functional status; neurological surgery outcomes; vascular disorders

$\mathrm{P}$ EDIATRIC brain arteriovenous malformations (bAVMs) are rare but account for nearly $50 \%$ of all sources of intracranial hemorrhage $(\mathrm{ICH})$ in the pediatric population, representing the most common cause of hemorrhagic stroke. ${ }^{7,11}$ Unfortunately, ICH due to a ruptured bAVM is associated with significant mortality and neurological morbidity, often culminating in clinical disability., ${ }^{2,16}$ Interestingly, pediatric patients seem to demonstrate superior outcomes in comparison with adult patients with similar lesions and presentations, although the mechanism underlying these superior outcomes is largely understudied and remains unknown..$^{21,23}$ It is important to precisely measure and accurately categorize disability associated with bAVM presentation and treatment to offer families prognosis and informed hopes for neurological recovery. Most series of pediatric bAVM clinical outcomes use the modified Rankin

ABBREVIATIONS AVM = arteriovenous malformation; bAVM = brain AVM; CNS = central nervous system; EVD = external ventricular drain; GCS = Glasgow Coma Scale; $\mathrm{GOS}=$ Glasgow Outcome Scale; HRQL = health-related quality of life; ICH = intracranial hemorrhage; $\mathrm{mRS}=$ modified Rankin Scale; PCPC = Pediatric Cerebral Performance Category; PedsQL = Pediatric Quality of Life Inventory; POPC = Pediatric Overall Performance Category; SAH = subarachnoid hemorrhage; TBI = traumatic brain injury; VP = ventriculoperitoneal; WFNS = World Federation of Neurosurgical Societies.

SUBMITTED February 22, 2016. ACCEPTED June 6, 2016.

INCLUDE WHEN CITING Published online August 19, 2016; DOI: 10.3171/2016.6.PEDS16103. 
Scale (mRS) to measure disability, despite a lack of validation in pediatric patients. Other common classification schemes used to categorize clinical outcomes include the Glasgow Outcome Scale (GOS), the Pediatric Cerebral Performance Category (PCPC) scale, and the Pediatric Overall Performance Category (POPC) scale. ${ }^{10}$ Yet none of these assays are equipped to capture a full spectrum of "disability"- defined by the World Health Organization as an umbrella term for impairments, activity limitations, and participation restrictions. ${ }^{29}$

We retrospectively evaluated a pediatric bAVM cohort treated with multimodality therapies utilizing the Pediatric Quality of Life Inventory (PedsQL 4.0), the most commonly employed health-related quality of life (HRQL) assay in the pediatric literature. ${ }^{25-27}$ The goals of the study were 1) to determine whether PedsQL scores were comparable to conventional metrics for clinical outcomes (i.e., $\mathrm{mRS}, \mathrm{GOS}, \mathrm{POPC}$, and PCPC scores) and 2) to interrogate various bAVM-related parameters (i.e., location, presentation, Spetzler-Martin grade, etc.) as potential predictors of poor HRQL scores.

\section{Methods}

After obtaining institutional review board approval, we retrospectively reviewed our experience with treated bAVMs from January 2005 to December 2012. We identified 46 pediatric patients treated with multimodality therapy, previously reported. ${ }^{18}$ We excluded 1 deceased child, 2 children who had previous bAVM-related treatment at outside institutions, and 9 children who were lost to follow-up, leaving 34 eligible patients. We successfully contacted the parents of 26 patients (76\%) and administered the parent proxy of the PedsQL 4.0-a well-validated 23-item questionnaire that assesses pediatric HRQL in 4 domains: physical, emotional, social, and school. Each question generates a score ranging from 0 to 4 , with lower values representing less disability. Total PedsQL scores were then inversely converted to a HRQL score, ranging from 0 to 100, with higher scores indicating better outcomes. There are multiple customized PedsQL surveys available for different age groups, and in most cases both a patient and parent survey are available. We opted to limit our survey strictly to parent reports, given the limitations associated with telephone interviews and unknown, unpredictable levels of cognitive injury associated with prior hemorrhage. We interviewed whichever parent was most readily available over the phone. Nonetheless, in 2 instances when a parent was unavailable and the patient's current age was greater than 18 years, we interviewed the patient. A 4.5-point change in the HRQL score for parent proxy report has previously been determined to represent clinical significance..$^{24,26}$ PedsQL, mRS, POPC, and PCPC scores were considered primary outcomes. We investigated a number of risk factors suspected to correlate with functional outcome scores. These included 1) features associated with presentation (age, need for external ventricular drain [EVD], hemorrhage, seizure, headache, neurological deficit, incidental, Glasgow Coma Scale [GCS] total and motor scores, World Federation of Neurosurgical Societies [WFNS] subarachnoid hemorrhage [SAH] grades, and
mRS scores), 2) anatomical features (type of hemorrhage, clot volume, Spetzler-Martin grade, specific brain location, nidus volume, deep venous drainage, and deep brain location), 3) hospital course (treatment modality, number of interventions, permanent CSF diversion, both total and intensive care unit length of stay, "cured" status, complications, and disposition facility), and 4) metrics for disability (special education, chronic daily medication use, and corrective devices). We also controlled for a number of variables that were not hypothesized to affect outcomes, including sex, race, total household income, parent education level, parent interviewed, "only child" status, and lone-parent household.

Univariate statistical analysis was conducted using the Kruskal-Wallis test and Spearman correlation as appropriate. Multivariate analysis was carried out using multiple linear regression with a stepwise algorithm that selected for statistically significant $(\mathrm{p}<0.05)$ main and interaction effects.

Finally, we reviewed the literature regarding both 1) long-term clinical outcomes in pediatric bAVM and 2) prior utilization of the PedsQL in pediatric neurological injuries to understand the context of HRQL impairments in pediatric $\mathrm{bAVM}$.

\section{Results}

We were able to contact a parent or patient over the phone in $26(76 \%)$ of the 34 pediatric bAVM cases that we identified as involving patients eligible for this study The demographic and clinical characteristics of this 26-patient cohort are summarized in Table 1 . The average PedsQL HRQL score was $71 \pm 24$, with an average age at diagnosis of 12.5 years, and an average follow-up period of 6.8 years (Table 2). Seventeen patients $(65 \%)$ had presented with hemorrhage, and $4(15 \%)$ with seizures. There were 5 Spetzler-Martin Grade I, 4 Grade II, 10 Grade III, 4 Grade IV, and 3 Grade V lesions. Five patients were treated with surgical intervention exclusively, 6 with radiation therapy, and 14 with multimodality therapy. One patient with a Grade V bAVM that was not amenable to cure by either surgery or radiosurgery was treated with targeted endovascular embolization. At most recent follow-up, "radiographic obliteration" had been achieved in $18(69 \%)$ of the 26 cases.

PedsQL correlated strongly and at a statistically significant level $(p<0.001)$ with the other primary metrics (Fig. 1). This was largely true as well for the subcategories within the PedsQL survey, with the exceptions that mRS and POPC did not seem to correlate with "school" measured disability (Table 3).

Of all the variables of interest that were investigated, univariate analysis demonstrated the following to reach a statistically significant correlation with PedsQL score: special education, chronic medication use, corrective devices, placement of an EVD on presentation, placement of a ventriculoperitoneal (VP) shunt, complications, and nidus volume (Table 2). Additionally, cured status showed a trend toward significant correlation with PedsQL score $(\mathrm{p}=0.075)$ and a statistically significant correlation with the other 4 investigated functional metrics, so it was in- 
TABLE 1. Cohort of patients included in analysis

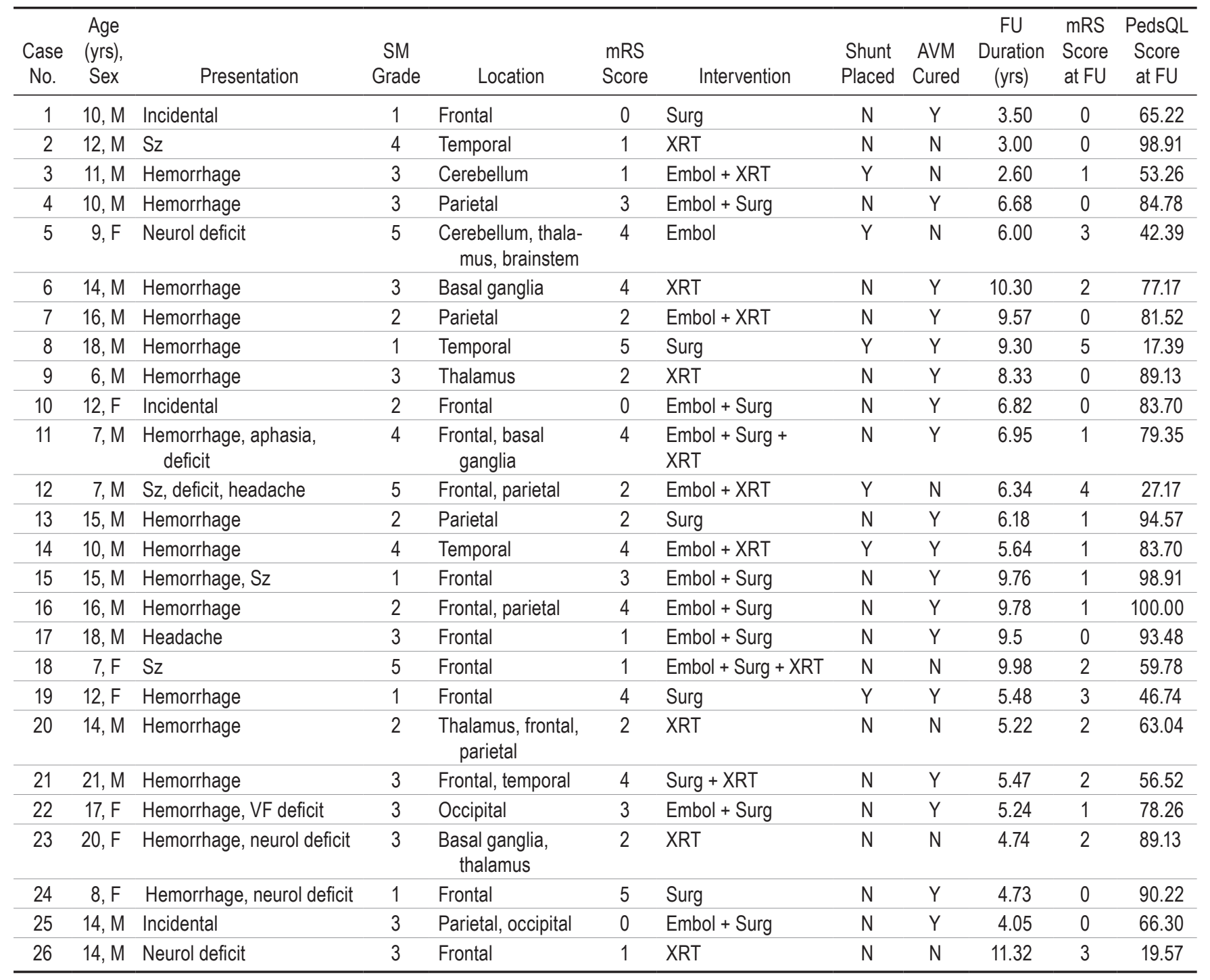

Embol = embolization; FU = follow-up; neurol = neurological; SM = Spetzler-Martin; Surg = surgery; Sz = seizure(s); VF = visual field; XRT = radiation therapy.

cluded in multivariate modeling (Fig. 2). Total length of hospital stay was also included in the model. Features that were suspected to correlate with worse outcomes are noted in Table 2. Subsequent multivariate modeling validated special education, corrective devices, and cure status to all correlate independently with PedsQL (Table 4). Additionally, the parent interviewed was significantly related to PedsQL, with mothers generating significantly lower PedsQL HRQL scores than fathers (61 vs 86 on average). Statistically significant risk factors for undergoing placement of a VP shunt included lower GCS motor scores on admission ( $p=0.042)$, cerebellar location $(p=0.046)$, and nidus volume ( $p=0.017)$. Spetzler-Martin grade, deep venous drainage, and deep brain location were all unrelated to PedsQL. Treatment modality also was not found to have a statistically significant affect on clinical outcomes at follow-up. Specific brain location did not predict PedsQL scores in any location (i.e., frontal, temporal, parietal, occipital, cerebellar, basal ganglia, thalamus, or brainstem).
Nonetheless, Fig. 3 depicts the adjusted (after accounting for parent interviewed) difference in PedsQL score with involvement in various parts of cortex (not statistically significant).

\section{Discussion}

It is important to accurately assess a child's comprehensive health, functional status, and disability to 1) characterize patterns of neurocognitive injury in order to guide resource allocation, 2) guide discussions with family members regarding expectations for both initial injuries and extent of anticipated recovery, and 3) implement treatment (i.e., neuropsychological therapy) to help strengthen focal areas of weakness in cognitive performance. Prior reports of clinical outcomes for pediatric bAVM patients, including one recently published by our group, ${ }^{18}$ are limited to utilizing the $\mathrm{mRS}$, which, despite ample validation in the adult stroke population, unfortunately limits the extent 
TABLE 2. Demographics of the cohort, with associated mean HRQL scores

\begin{tabular}{|c|c|c|c|c|c|}
\hline \multirow[b]{2}{*}{ Variable } & \multirow[b]{2}{*}{ No. of Pts (\%) } & \multirow{2}{*}{$\begin{array}{c}\text { Mean HRQL } \\
\text { Score (SD) }\end{array}$} & \multirow[b]{2}{*}{ Significance $†$} & \multicolumn{2}{|c|}{ For Quantitative Variables } \\
\hline & & & & Mean (SD) & Rho \\
\hline Special education & & & $0.008 \S$ & & \\
\hline No & $12(46 \%)$ & $84(18)$ & & & \\
\hline Yes & $14(54 \%)$ & $60(24)$ & & & \\
\hline Chronic medication Tx & & & $0.020 \S$ & & \\
\hline No & $16(62 \%)$ & $80(17)$ & & & \\
\hline Yes & $10(38 \%)$ & $55(28)$ & & & \\
\hline Corrective devices & & & $<0.001 \S$ & & \\
\hline No & $18(69 \%)$ & $83(14)$ & & & \\
\hline Yes & $8(31 \%)$ & $44(22)$ & & & \\
\hline Age at surg & & & $0.462 \rrbracket$ & $12.5(3.4)$ & 0.15 \\
\hline$<10 \mathrm{yrs}$ & $7(27 \%)$ & $68(25)$ & & & \\
\hline $10-14$ yrs & $11(42 \%)$ & $67(22)$ & & & \\
\hline$\geq 15 \mathrm{yrs}$ & $8(31 \%)$ & $79(29)$ & & & \\
\hline Time since surg & & & 0.892 & $6.8(2.5)$ & 0.03 \\
\hline$<6 \mathrm{yrs}$ & $11(42 \%)$ & $72(17)$ & & & \\
\hline $6-8$ yrs & $7(27 \%)$ & $72(26)$ & & & \\
\hline$\geq 9$ yrs & $8(31 \%)$ & $68(34)$ & & & \\
\hline Cure status & & & $0.075 \S$ & & \\
\hline No & $8(31 \%)$ & $57(28)$ & & & \\
\hline Yes & $18(69 \%)$ & $77(21)$ & & & \\
\hline Sex & & & 0.664 & & \\
\hline Female & $7(27 \%)$ & $70(20)$ & & & \\
\hline Male & $19(73 \%)$ & $71(26)$ & & & \\
\hline Race & & & 0.936 & & \\
\hline Alaskan/Native American & $3(12 \%)$ & $56(33)$ & & & \\
\hline White & $14(54 \%)$ & $72(23)$ & & & \\
\hline Hispanic & $4(15 \%)$ & $70(35)$ & & & \\
\hline Black & $2(8 \%)$ & $83(2)$ & & & \\
\hline Asian & $2(8 \%)$ & $71(34)$ & & & \\
\hline Hawaiian & $1(4 \%)$ & $77(-)$ & & & \\
\hline Household income & & & 0.293 & $\$ 82 \mathrm{~K}(\$ 50 \mathrm{~K}) \ddagger$ & -0.30 \\
\hline$<\$ 25 \mathrm{~K}$ & $1(4 \%)$ & $99(-)$ & & & \\
\hline$\$ 25-50 \mathrm{~K}$ & $9(35 \%)$ & $80(15)$ & & & \\
\hline$\$ 50-75 K$ & $5(19 \%)$ & $56(30)$ & & & \\
\hline$\$ 75-100 K$ & $3(12 \%)$ & $76(15)$ & & & \\
\hline$>\$ 100 \mathrm{~K}$ & $8(31 \%)$ & $64(29)$ & & & \\
\hline Parents' education & & & 0.205 & & \\
\hline Less than high school & $1(4 \%)$ & $99(-)$ & & & \\
\hline High school & $9(35 \%)$ & $61(28)$ & & & \\
\hline Partial college & $1(4 \%)$ & $20(-)$ & & & \\
\hline Full college degree & $9(35 \%)$ & 75 (19) & & & \\
\hline Graduate degree & $6(23 \%)$ & $82(13)$ & & & \\
\hline Parents' no. of yrs of education & & & & $15(2.9)$ & 0.16 \\
\hline Parent interviewed & & & $0.036 \S$ & & \\
\hline Mother & $15(58 \%)$ & $61(26)$ & & & \\
\hline Father & $9(35 \%)$ & $86(12)$ & & & \\
\hline Patient & $2(8 \%)$ & $78(16)$ & & & \\
\hline Only child & & & 0.434 & & \\
\hline No & $22(85 \%)$ & $69(26)$ & & & \\
\hline Yes & $4(15 \%)$ & $82(11)$ & & & \\
\hline Lone parent & & & 0.58 & & \\
\hline No & $21(81 \%)$ & $69(26)$ & & & \\
\hline Yes & $5(19 \%)$ & 79 (15) & & & \\
\hline
\end{tabular}


» CONTINUED FROM PAGE 614

TABLE 2. Demographics of the cohort, with associated mean HRQL scores

\begin{tabular}{|c|c|c|c|c|c|}
\hline \multirow[b]{2}{*}{ Variable } & \multirow[b]{2}{*}{ No. of Pts (\%) } & \multirow{2}{*}{$\begin{array}{c}\text { Mean HRQL } \\
\text { Score (SD) }\end{array}$} & \multirow[b]{2}{*}{ Significance $†$} & \multicolumn{2}{|c|}{ For Quantitative Variables } \\
\hline & & & & Mean (SD) & Rho \\
\hline Hemorrhage & & & $0.269 \rrbracket$ & & \\
\hline No & $9(35 \%)$ & $62(28)$ & & & \\
\hline Yes & $17(65 \%)$ & $76(22)$ & & & \\
\hline Sz & & & 0.67 & & \\
\hline No & $22(85 \%)$ & $71(23)$ & & & \\
\hline Yes & $4(15 \%)$ & $71(35)$ & & & \\
\hline Headache & & & 0.626 & & \\
\hline No & $20(77 \%)$ & $72(25)$ & & & \\
\hline Yes & $6(23 \%)$ & $67(25)$ & & & \\
\hline Neurol deficits & & & 0.583 & & \\
\hline No & $7(27 \%)$ & $70(16)$ & & & \\
\hline Yes & $19(73 \%)$ & $71(27)$ & & & \\
\hline Incidental & & & 0.81 & & \\
\hline No & $23(88 \%)$ & $71(26)$ & & & \\
\hline Yes & $3(12 \%)$ & $72(10)$ & & & \\
\hline Admission GCS score & & & 0.306 & $12.1(3.8)$ & -0.21 \\
\hline $3-8$ & $6(23 \%)$ & $66(31)$ & & & \\
\hline $9-12$ & $3(12 \%)$ & $89(10)$ & & & \\
\hline $13-15$ & $17(65 \%)$ & $69(23)$ & & & \\
\hline Admission GCS motor score & & & 0.26 & $5.5(1.2)$ & 0.23 \\
\hline $1-5$ & $5(19 \%)$ & $59(29)$ & & & \\
\hline 6 & $21(81 \%)$ & $74(23)$ & & & \\
\hline WFNS grade & & & 0.277 & $2.6(1.5)^{* *}$ & 0.22 \\
\hline I & $11(42 \%)$ & $61(25)$ & & & \\
\hline$\|$ & $1(4 \%)$ & $82(-)$ & & & \\
\hline III & $5(19 \%)$ & $85(7)^{\prime}$ & & & \\
\hline IV & $6(23 \%)$ & $83(20)$ & & & \\
\hline V & $3(12 \%)$ & $55(36)$ & & & \\
\hline mRS score & & & 0.888 & $2.5(1.6)$ & -0.03 \\
\hline 0 & $3(12 \%)$ & $72(10)$ & & & \\
\hline 1 & $5(19 \%)$ & $65(32)$ & & & \\
\hline 2 & $6(23 \%)$ & $74(25)$ & & & \\
\hline 3 & $3(12 \%)$ & $87(11)$ & & & \\
\hline 4 & $7(27 \%)$ & $69(21)$ & & & \\
\hline 5 & $2(8 \%)$ & $54(51)$ & & & \\
\hline Type of clot & & & 0.358 & & \\
\hline None & $9(35 \%)$ & $62(28)$ & & & \\
\hline $\mathrm{IPH}$ & $6(23 \%)$ & $68(29)$ & & & \\
\hline $\mathrm{IPH}+\mathrm{IVH}$ & $11(42 \%)$ & $79(17)$ & & & \\
\hline Volume of clot $\left(\mathrm{cm}^{3}\right)$ & & & $0.609^{* *}$ & $15.8(19.7)$ & 0.11 \\
\hline SM grade & & & $0.273 \pi$ & $2.8(1.3) \dagger \dagger$ & -0.22 \\
\hline I & $5(19 \%)$ & $64(33)$ & & & \\
\hline$\|$ & $4(15 \%)$ & $90(9)^{\prime}$ & & & \\
\hline III & $10(38 \%)$ & $71(23)$ & & & \\
\hline IV & $4(15 \%)$ & $81(15)$ & & & \\
\hline V & $3(12 \%)$ & $43(16)$ & & & \\
\hline Frontal & & & $0.700 \rrbracket$ & & \\
\hline No & $13(50 \%)$ & $74(23)$ & & & \\
\hline Yes & $13(50 \%)$ & $68(26)$ & & & \\
\hline Temporal & & & $0.722 \pi$ & & \\
\hline No & $22(85 \%)$ & $72(23)$ & & & \\
\hline Yes & $4(15 \%)$ & $64(36)$ & & & \\
\hline
\end{tabular}


» CONTINUED FROM PAGE 615

TABLE 2. Demographics of the cohort, with associated mean HRQL scores

\begin{tabular}{|c|c|c|c|c|c|}
\hline \multirow[b]{2}{*}{ Variable } & \multirow[b]{2}{*}{ No. of Pts (\%) } & \multirow{2}{*}{$\begin{array}{c}\text { Mean HRQL } \\
\text { Score (SD) }\end{array}$} & \multirow[b]{2}{*}{ Significance† } & \multicolumn{2}{|c|}{ For Quantitative Variables } \\
\hline & & & & Mean (SD) & Rho \\
\hline Parietal & & & $0.544 \pi$ & & \\
\hline No & $19(73 \%)$ & $70(25)$ & & & \\
\hline Yes & $7(27 \%)$ & $74(25)$ & & & \\
\hline Occipital & & & $0.773 \rrbracket$ & & \\
\hline No & $24(92 \%)$ & $71(25)$ & & & \\
\hline Yes & $2(8 \%)$ & $72(8)$ & & & \\
\hline Cerebellar & & & $0.102 \pi$ & & \\
\hline No & $24(92 \%)$ & $73(24)$ & & & \\
\hline Yes & $2(8 \%)$ & $48(8)$ & & & \\
\hline Basal ganglia & & & $0.688 \pi$ & & \\
\hline No & $23(88 \%)$ & $69(26)$ & & & \\
\hline Yes & $3(12 \%)$ & $82(6)$ & & & \\
\hline Thalamus & & & $0.887 \rrbracket$ & & \\
\hline No & $22(85 \%)$ & $71(25)$ & & & \\
\hline Yes & $4(15 \%)$ & $71(23)$ & & & \\
\hline Brainstem & & & $0.205 \rrbracket$ & & \\
\hline No & $25(96 \%)$ & $72(24)$ & & & \\
\hline Yes & $1(4 \%)$ & $42(-)$ & & & \\
\hline Nidus vol $\left(\mathrm{cm}^{3}\right)$ & & & $0.010^{* *} \S$ & $15.3(28.2)$ & -0.51 \\
\hline Deep venous drainage & & & $0.616 \pi$ & & \\
\hline No & $10(38 \%)$ & $74(24)$ & & & \\
\hline Yes & $16(62 \%)$ & $69(25)$ & & & \\
\hline Deep brain location & & & $0.855 \rrbracket$ & & \\
\hline No & $20(77 \%)$ & $70(26)$ & & & \\
\hline Yes & $6(23 \%)$ & $73(18)$ & & & \\
\hline EVD placed & & & $0.049 \S$ & & \\
\hline No & $17(65 \%)$ & $77(22)$ & & & \\
\hline Yes & $9(36 \%)$ & $57(25)$ & & & \\
\hline VP shunt & & & $0.003 \S$ & & \\
\hline No & $20(77 \%)$ & $78(19)$ & & & \\
\hline Yes & $6(23 \%)$ & $45(23)$ & & & \\
\hline Surg & & & 0.364 & & \\
\hline No & $11(42 \%)$ & $66(27)$ & & & \\
\hline Yes & $15(58 \%)$ & $74(23)$ & & & \\
\hline Embolization & & & 0.662 & & \\
\hline No & $12(46 \%)$ & $67(28)$ & & & \\
\hline Yes & $14(54 \%)$ & $74(21)$ & & & \\
\hline XRT & & & 0.317 & & \\
\hline No & $13(50 \%)$ & $74(25)$ & & & \\
\hline Yes & $13(50 \%)$ & $68(24)$ & & & \\
\hline Multiple Tx & & & 0.56 & & \\
\hline No & $12(46 \%)$ & $66(29)$ & & & \\
\hline Yes & $14(54 \%)$ & $75(20)$ & & & \\
\hline Complications & & & $0.023 \S$ & & \\
\hline No & $21(81 \%)$ & $77(20)$ & & & \\
\hline Yes & $5(19 \%)$ & $46(28)$ & & & \\
\hline Total LOS & & & $0.085^{* *} \S$ & $13.1(11.2)$ & -0.34 \\
\hline ICU LOS & & & $0.16^{* *}$ & $9.2(9.1)$ & -0.28 \\
\hline
\end{tabular}


» CONTINUED FROM PAGE 616

TABLE 2. Demographics of the cohort, with associated mean HRQL scores

\begin{tabular}{|c|c|c|c|c|c|}
\hline \multirow[b]{2}{*}{ Variable } & \multirow[b]{2}{*}{ No. of Pts (\%) } & \multirow{2}{*}{$\begin{array}{c}\text { Mean HRQL } \\
\text { Score (SD) }\end{array}$} & \multirow[b]{2}{*}{ Significance $†$} & \multicolumn{2}{|c|}{ For Quantitative Variables } \\
\hline & & & & Mean (SD) & Rho \\
\hline Disposition & & & 0.411 & & \\
\hline Outpatient procedure & $1(4 \%)$ & $77(-)$ & & & \\
\hline Home & $13(50 \%)$ & $74(21)$ & & & \\
\hline Rehab & $11(42 \%)$ & $71(26)$ & & & \\
\hline SNF & $1(4 \%)$ & $17(-)$ & & & \\
\hline
\end{tabular}

$\mathrm{ICU}=$ intensive care unit; IPH = intraparenchymal hemorrhage; IVH = intraventricular hemorrhage; $\mathrm{LOS}$ = length of stay; pts = patients; Rehab = rehabilitation facility; SNF = skilled nursing facility; $\mathrm{Tx}$ = treatment(s).

* Values are $p$ values unless otherwise indicated. Boldface type indicates statistical significance.

$\dagger$ Based on univariate analysis.

$\ddagger$ Approximate mean.

$\S$ Was considered as a potential predictor in the multivariate modeling.

I Was expected to be a significant univariate predictor of HRQL but was not.

${ }_{* *}$ Rho value. For these analyses, the volume or LOS was normalized and compared to HRQL to determine whether there is a relationship between the 2 data sets as a whole.

t† Converted to Arabic numerals for purposes of statistical analysis.

of assessment in children. Similarly, the GOS, POPC, and PCPC scales fail to grasp "the big picture" of collective disability. This is particularly concerning in the pediatric population, as neurological insults may occur at various stages of brain development, resulting in a wide array of disability and effects on HRQL. ${ }^{2}$ There are mixed reports in the literature regarding the timing of brain injury, on one hand arguing for enhanced recovery in the setting of

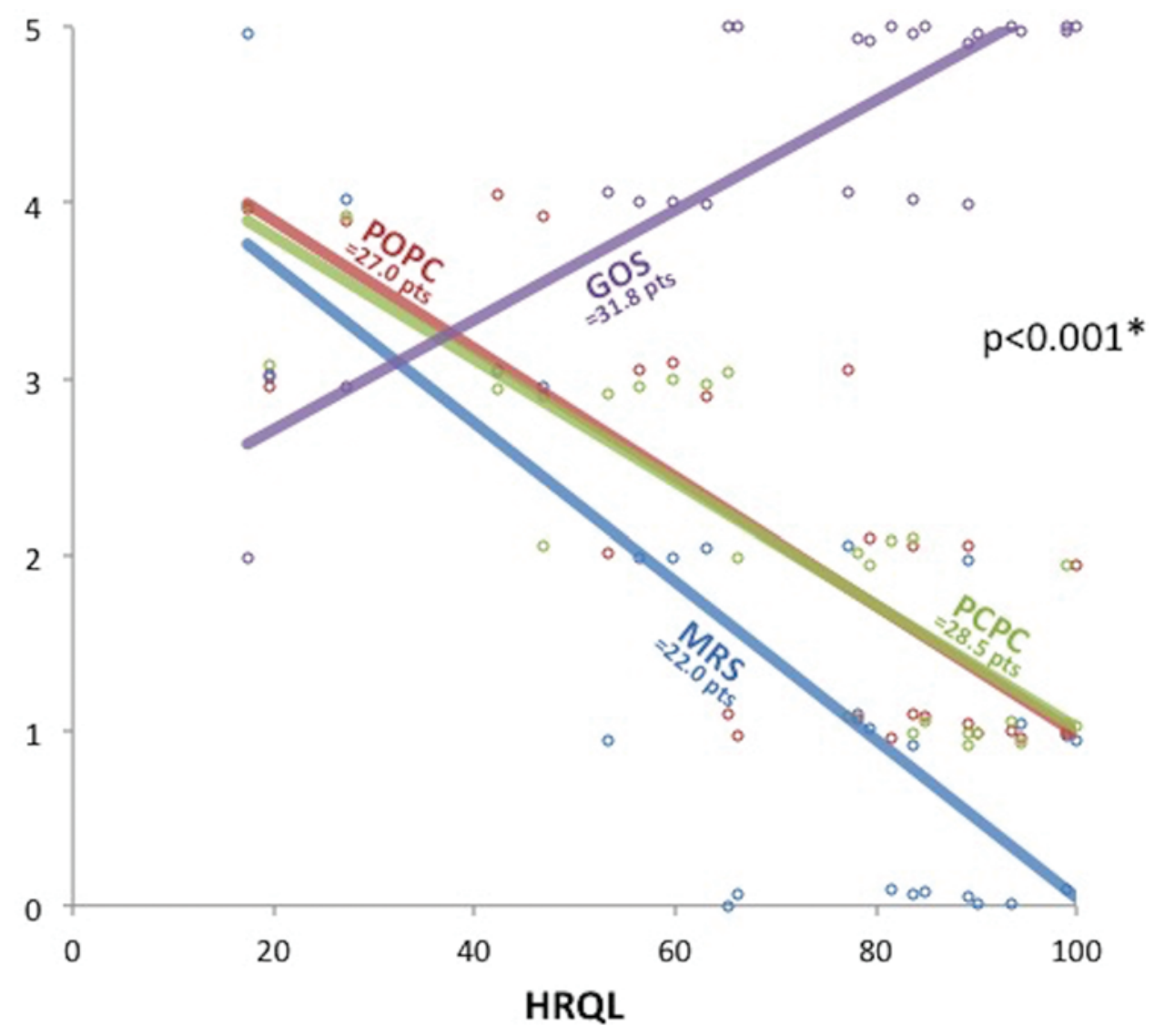

FIG. 1. Regression analysis of other primary metrics (mRS in blue, GOS in purple, POPC in red, and PCPC in green) as a function of PedsQL HRQL (x-axis). All correlations had a p value $<0.001$, indicating significant correlation. Slope values are indicated on curves to quantify the correlative differences. For example, each increase in the mRS score correlated with 22 points (pts) less in the PedsQL. Figure is available in color online only. 
TABLE 3. Spearman correlation comparing overall PedsQL scores as well as subcomponents to conventional metrics for functional outcomes*

\begin{tabular}{|c|c|c|c|c|c|c|c|c|}
\hline \multirow{2}{*}{$\begin{array}{l}\text { PedsQL } \\
\text { Component }\end{array}$} & \multicolumn{2}{|c|}{ mRS Score } & \multicolumn{2}{|c|}{ POPC Score } & \multicolumn{2}{|c|}{ PCPC Score } & \multicolumn{2}{|c|}{ GOS Score } \\
\hline & Rho & $p$ Value & Rho & p Value & Rho & p Value & Rho & $p$ Value \\
\hline Overall score & -0.67 & 0.001 & -0.74 & $<0.001$ & -0.84 & $<0.001$ & 0.79 & $<0.001$ \\
\hline \multicolumn{9}{|l|}{ Subscores } \\
\hline Physical & -0.76 & 0.001 & -0.78 & $<0.001$ & -0.66 & $<0.001$ & 0.85 & $<0.001$ \\
\hline Emotional & -0.45 & 0.020 & -0.52 & 0.007 & -0.63 & 0.001 & 0.65 & $<0.001$ \\
\hline Social & -0.58 & 0.002 & -0.63 & 0.001 & -0.83 & $<0.001$ & -0.65 & $<0.001$ \\
\hline School & -0.30 & 0.139 & -0.38 & 0.053 & -0.82 & $<0.001$ & 0.46 & 0.019 \\
\hline
\end{tabular}

* Note that the "school" HRQL subcomponent did not correlate with mRS scores at a significant level.

pediatric brain injury due to cortical plasticity, ${ }^{14,21,23}$ further supported with reports of superior outcomes in neonatal stroke compared with childhood stroke. ${ }^{8}$ Yet, others have reported poor motor and neurocognitive outcomes of brain injuries prior to 1 or 2 years of age.,17

The Pediatric Quality of Life Inventory (PedsQL 4.0) is the most widely employed survey in assessing HRQL in the pediatric population, with an acceptable validity, reliability, and tremendous feasibility. ${ }^{25,27}$ The survey consists of 23 questions targeting 4 domains: physical, emotional, social, and school. Indeed, it has been validated in a number of pediatric brain pathologies, including ischemic stroke,,$^{12}$ traumatic brain injury (TBI), ${ }^{20,24}$ and brain tumors. ${ }^{3,6,15}$ Healthy children score an average of 82.3 ,
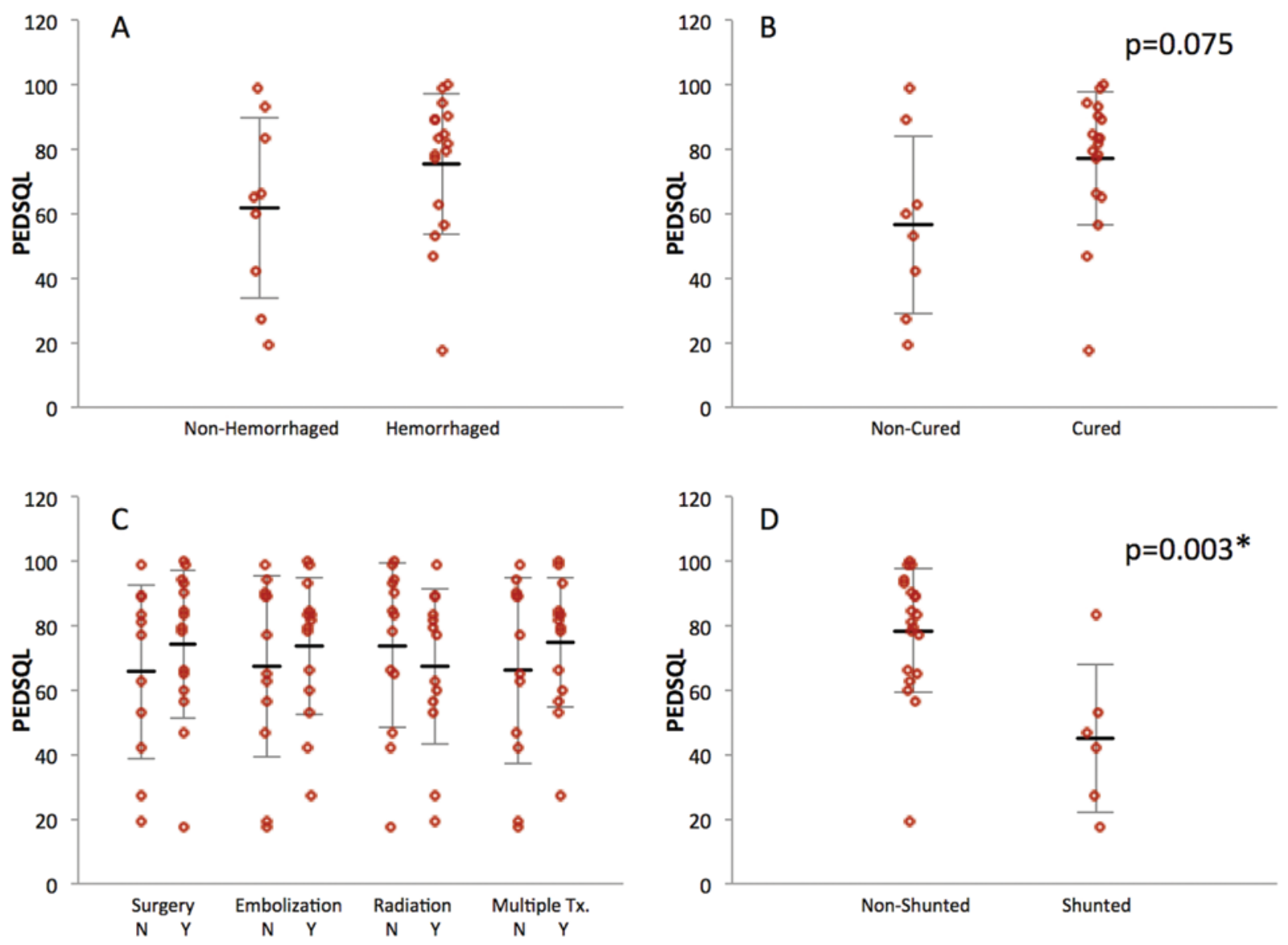

FIG. 2. Dot plots of PedsQL scores at most recent follow-up, with Mann-Whitney significance values reported where notable. A: Hemorrhaged versus unruptured bAVMs. There was no statistically significant difference between the 2 populations, although there was a trend for patients with unruptured lesions to have lower HRQL scores at follow-up. B: On average, patients with angiographically cured lesions had higher $\mathrm{HRQL}$ scores, and although not statistically significant, this difference trended toward significance $(p=0.075)$. C: Treatment modality employed did not have a statistical effect on HRQL scores. D: The presence of a VP shunt showed a statistically significant correlation with lower HRQL scores after univariate analysis. Figure is available in color online only. 
TABLE 4. Multivariate modeling*

\begin{tabular}{lrrrrr}
\hline \multicolumn{1}{c}{ Predicting HRQL } & $\mathrm{B}$ & $\mathrm{SE}$ & $\mathrm{t}$ & $\mathrm{p}$ Value & \multicolumn{1}{c}{$95 \% \mathrm{Cl}$} \\
\hline (Constant) & 86.02 & 7.28 & 11.82 & $<0.001$ & 70.79 to 101.24 \\
\hline Special education & -12.13 & 5.17 & -2.35 & 0.030 & -22.94 to -1.31 \\
\hline Chronic medication Tx & 1.43 & 8.96 & 0.16 & 0.875 & -17.31 to 20.18 \\
\hline Corrective devices & -20.42 & 6.97 & -2.93 & 0.009 & -35.00 to -5.84 \\
\hline Cure status & 16.33 & 7.79 & 2.10 & 0.050 & 0.03 to 32.62 \\
\hline Mother interviewed & -15.57 & 5.55 & -2.81 & 0.011 & -27.18 to -3.96 \\
\hline ChrMed * CureStat & -22.94 & 10.87 & -2.11 & 0.048 & -45.70 to -0.19 \\
\hline
\end{tabular}

ChrMed $=$ chronic medication treatment; CureStat $=$ cure status .

* $R^{2}$ 0.805. Adjusted $R^{2}$ 0.743. Special education, corrective devices, and cure status form a strong subset of predictors of $H R Q L$, which is improved further by adjusting for the interview source and including an interaction effect between cure status and chronic medication use. This model explained $80 \%$ of the variability in $\mathrm{HRQL}$.

whereas the average score for children with chronic medical conditions is 73.1 .25

Children in our series showed HRQL scores (71 on average) similar to children with other chronic conditions such as asthma or diabetes, as well as other pediatric central nervous system (CNS) pathologies (Table 5). We identified special education, corrective devices, and the lack of radiographic obliteration as all statistically correlating with worse PedsQL outcome scores. Although larger nidus volume correlated with worse outcomes in univariate analysis, this correlation was not sustained in multivari- ate modeling. Interestingly, none of the classic anatomical features associated with increased risk of hemorrhage (i.e., hemorrhage on presentation, deep venous drainage, and deep brain location) or specific brain location played a statistically significant role in predicting worse outcomes. However, infratentorial lesions (i.e., brainstem and cerebellar lesions) seemed to show worse outcomes, with parietal lesions (the least eloquent) showing the best (see Fig. 3). Table 6 summarizes all prior reported studies that have assessed "clinical outcomes," including most recently a report from our institution. Surprisingly, there is not a

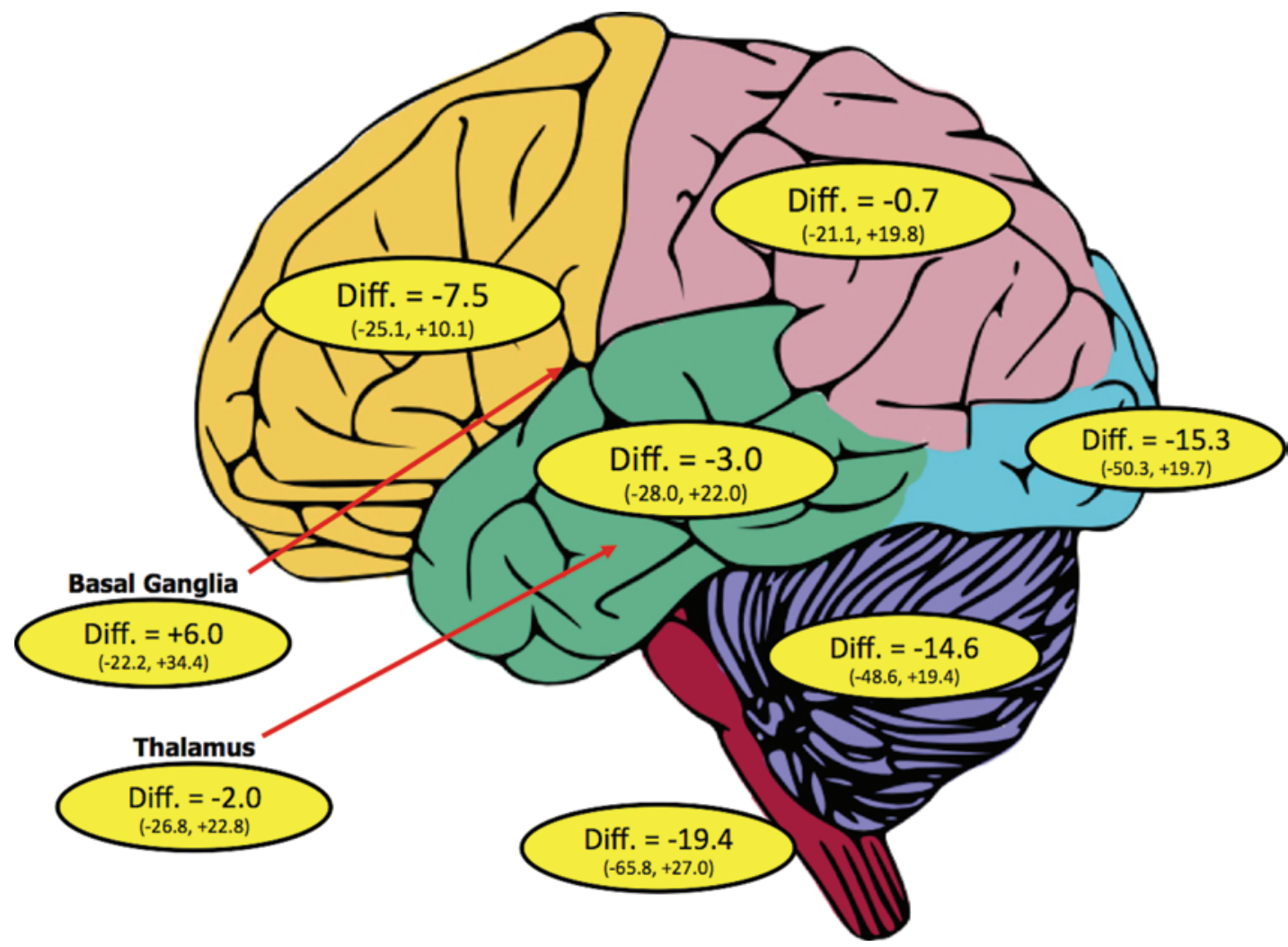

FIG. 3. Map of brain location and representative "delta" values (Diff.) for PedsQL HRQL scores after adjusting for parent interviewed in multivariate modeling. None of the values reached statistical significance. The upper and lower bounds of the $95 \%$ confidence intervals are listed in parentheses for each location. Note that although the differences were not statistically significant, parietal bAVMs showed the least negative impact (-0.7 points) and posterior fossa lesions showed the most substantial negative impacts to $H R Q L$ scores (-19.4 points in brainstem lesions and -14.6 points in cerebellar lesions). Figure is available in color online only. 
TABLE 5. Prior reports of PedsQL HRQL scores in CNS-related pathologies, including TBI, brain tumors, stroke, and spina bifida

\begin{tabular}{|c|c|c|c|c|c|}
\hline Study & $\begin{array}{l}\text { Total No. of } \\
\text { Pts }\end{array}$ & Pathology & Subgroup & $\begin{array}{l}\text { Avg FU Interval from } \\
\text { Dx/Injury (yrs) }\end{array}$ & $\begin{array}{l}\text { PedsQL HRQL } \\
\text { Score }(0-100)\end{array}$ \\
\hline \multirow[t]{5}{*}{ Bhat et al., $2005^{*}$} & \multirow[t]{5}{*}{134} & \multirow[t]{5}{*}{ Brain tumors/Tx } & No Tx & 4.26 & 73.03 \\
\hline & & & Surg & 4.26 & 75.21 \\
\hline & & & RT + Surg & 4.26 & 52.57 \\
\hline & & & RT + Chemo + Surg & 4.26 & 70.27 \\
\hline & & & Chemo + Surg & 4.26 & 72.18 \\
\hline \multirow[t]{5}{*}{ Bhat et al., 2005* } & \multirow[t]{5}{*}{134} & \multirow[t]{5}{*}{ Brain tumors/pathology } & LGG & 4.26 & 75.36 \\
\hline & & & Medulloblastoma/PNET & 4.26 & 65.1 \\
\hline & & & Malignant glioma & 4.26 & 63.96 \\
\hline & & & Germ cell tumor & 4.26 & 74.36 \\
\hline & & & Other low-grade lesion & 4.26 & 61.5 \\
\hline \multirow[t]{6}{*}{ Rivara et al., 2012} & \multirow[t]{6}{*}{766} & \multirow[t]{6}{*}{ TBI } & Mild TBI w/ normal head CT & 3 & 82 \\
\hline & & & Mild TBI w/ skull fracture & 3 & 86.3 \\
\hline & & & Mild TBI w/ ICH & 3 & 83.5 \\
\hline & & & Mod TBI & 3 & 71.3 \\
\hline & & & Severe TBI & 3 & 67.2 \\
\hline & & & Isolated arm injury & 3 & 85.5 \\
\hline \multirow[t]{2}{*}{ Kuhlthau et al., $2012^{*}$} & \multirow[t]{2}{*}{142} & \multirow{2}{*}{$\begin{array}{l}\text { Brain tumors treated } \mathrm{w} / \\
\text { proton beam }\end{array}$} & Pre-Tx & 0 & 67 \\
\hline & & & Post-Tx & 3 & 76.5 \\
\hline \multirow[t]{5}{*}{ Kuhlthau et al., 2012* } & \multirow[t]{5}{*}{142} & \multirow{5}{*}{$\begin{array}{l}\text { Brain tumors treated } \mathrm{w} / \\
\text { proton beam }\end{array}$} & Medulloblastoma/PNET & 0 & 57.8 \\
\hline & & & Ependymoma/HGG & 0 & 69.8 \\
\hline & & & LGG & 0 & 71.5 \\
\hline & & & Other low-grade tumor & 0 & 78 \\
\hline & & & Germ cell/germinoma & 0 & 63.5 \\
\hline Ramachnadra et al., 2013 & 33 & Spina bifida & NA & NA & 59.9 \\
\hline \multirow[t]{3}{*}{ Ghotra et al., 2015} & \multirow[t]{3}{*}{90} & \multirow[t]{3}{*}{ Ischemic stroke } & Perinatal stroke & 5.17 & 68.8 \\
\hline & & & Neonatal stroke & 5.17 & 80 \\
\hline & & & Childhood stroke & 4.58 & 66.1 \\
\hline \multirow[t]{3}{*}{ Bull et al., 2015} & \multirow[t]{3}{*}{104} & \multirow{3}{*}{$\begin{array}{l}\text { Posterior fossa brain } \\
\text { tumors }\end{array}$} & Medulloblastoma & 3 & 51.5 \\
\hline & & & Astrocytoma & 3 & 68.2 \\
\hline & & & Nontumor control† & 3 & 84.3 \\
\hline
\end{tabular}

Avg = average; chemo = chemotherapy; Dx = diagnosis; HGG = high-grade glioma; LGG = low-grade glioma; mod = moderate; $N A=$ not available; $P N E T=$ primitive neuroectodermal tumor; $\mathrm{RT}$ = radiation therapy.

* Studies listed more than once in order to account for separate subgroup analysis performed.

$\dagger$ Cohort of healthy children without a brain tumor.

consistently used metric present in all studies, although mRS was used in 4 of 7 studies. Furthermore, there is a lack of a consistent definition of "poor outcomes," with $m R S>2$ or MRS $\geq 2$ used in 3 of the studies. Using the defined "normal" PedsQL score of 82.3 with 4.5 points of change indicating clinical increment, $12(46.2 \%)$ of the 26 children in our case series had clinically significant reductions in HRQL scores, indicating disability.

Sanchez-Mejia et al..$^{21}$ showed that children treated with microsurgical resection fared better than adults in terms of neurological outcomes, including mRS score at follow-up, changes in mRS score, and mean mRS score, regardless of AVM anatomy, rupture rates, neurological condition at presentation, or treatment regimen (i.e., some patients had preoperative embolization). Our findings that anatomical features were unrelated to long-term outcomes further support this notion. Darsaut et al. ${ }^{7}$ presented a prospectively collected database from Stanford of 120 pediatric bAVMs over 24 years, treated with various combinations of radiosurgery, microsurgery, and endovascular techniques. They evaluated patients' conditions with the $\mathrm{mRS}$ at presenta- tion and a variety of follow-up times and concluded that left-sided lesions, poor preoperative mRS scores, and high Spetzler-Martin grades were all independent risk factors for poor clinical outcomes (mRS score $\geq 2$ ), with highgrade lesions strongly correlated with higher complication rates and hemorrhage rates. Although this study has quite impressive follow-up (mean 9.2 years), there was a remarkably high complication rate with treatment of highgrade (Grade IV and V) AVMs (28\% compared with 5\% in low-grade lesions), although this was accounted for in multivariate modeling. Wong et al. ${ }^{28}$ analyzed mRS clinical outcomes at 6 months and "most recently" in a series of 40 treated pediatric bAVMs. They found Spetzler-Martin grade, intracerebral hemorrhage score, WFNS SAH grade, and GCS scores all to be predictive of clinical outcome (mRS score) at 6 months. Finally, Gross et al..$^{13}$ presented nearly 20 years of the Boston Children's Hospital experience, including 117 patients treated with microsurgical resection; $54 \%$ of their cohort had an mRS score of 0 at a median follow-up period of 3.6 years, and $94 \%$ had an mRS score of 2 or less. Our cohort represents-in con- 
TABLE 6. Prior studies evaluating clinical outcomes in pediatric cases of brain AVMs, with metrics employed and criteria used

\begin{tabular}{|c|c|c|c|c|c|c|c|}
\hline Study & $\begin{array}{c}\text { No. } \\
\text { of } \\
\text { Pts }^{*}\end{array}$ & $\begin{array}{c}\text { Tx } \\
\text { Modalities }\end{array}$ & $\begin{array}{c}\text { FU } \\
(\mathrm{yrs}) \dagger\end{array}$ & Metrics Employed & $\begin{array}{l}\text { Definition of Poor } \\
\text { Outcome }\end{array}$ & $\begin{array}{l}\text { Risk Factors for Poor } \\
\text { Outcome via } \\
\text { Multivariate Analysis }\end{array}$ & $\begin{array}{c}\text { Add'l Risk Factors from } \\
\text { Univariate Analysis }\end{array}$ \\
\hline $\begin{array}{l}\text { Di Rocco et } \\
\text { al., } 2000\end{array}$ & 37 & $\begin{array}{l}\text { Surg + XRT } \\
\quad+\text { Embol }\end{array}$ & 5.4 & $\begin{array}{l}\text { "Excellent, fair, good, } \\
\text { dead" }\end{array}$ & NA & NA & $\begin{array}{l}\text { Hemorrhage or neurol } \\
\text { deficits at presentation }\end{array}$ \\
\hline $\begin{array}{l}\text { Sanchez- } \\
\text { Mejia et al., } \\
2006\end{array}$ & 32 & $\begin{array}{l}\text { Surg + XRT } \\
\quad+\text { Embol }\end{array}$ & 5.2 & $\begin{array}{l}\text { Final mRS score, change } \\
\text { in } \mathrm{mRS} \text { score }\end{array}$ & $\begin{array}{c}\text { mRS score }>2 \text {, living } \\
\text { dependently, neu- } \\
\text { rol deterioration }\end{array}$ & $\begin{array}{l}\text { Older age (>18 yrs), } \\
\text { large size, eloquent } \\
\text { location }\end{array}$ & NA \\
\hline $\begin{array}{l}\text { Wong \& Fong, } \\
2010\end{array}$ & 40 & $\begin{array}{l}\text { Surg + XRT } \\
\quad+\text { Embol }\end{array}$ & $8.4 \ddagger$ & $\begin{array}{l}\text { mRS score } 6 \text { mos post- } \\
\text { hemorrhage }\end{array}$ & NA & $\begin{array}{l}\text { Combined presentation } \\
\text { scale (including high } \\
\text { GCS score, sluggish } \\
\text { pupillary response, } \\
\text { focal neurol deficit) }\end{array}$ & $\begin{array}{l}\text { SM grade, ICH score, } \\
\text { WFNS-SAH grade, } \\
\text { GCS score }\end{array}$ \\
\hline $\begin{array}{l}\text { Singhal et al., } \\
2011\end{array}$ & 15 & Surg & 5.4 & $\begin{array}{l}\text { GOS, PCPC, POPC } \\
\text { scores }\end{array}$ & $\begin{array}{l}\text { Functional depen- } \\
\text { dence }\end{array}$ & NA & NA \\
\hline $\begin{array}{l}\text { Darsaut et al., } \\
2011\end{array}$ & 120 & $\begin{array}{l}\text { Surg + XRT } \\
\quad+\text { Embol }\end{array}$ & 9.2 & mRS score & $m R S$ score $\geq 2$ & $\begin{array}{l}\text { Baseline } m R S \geq 2, \text { It } \\
\text { side location, SM } \\
\text { Grade IV or V }\end{array}$ & $\begin{array}{l}\text { Associated aneurysm, } \\
\text { eloquent cortex, deep } \\
\text { location }\end{array}$ \\
\hline $\begin{array}{c}\text { Blauwblomme } \\
\text { et al., } 2014\end{array}$ & 103 & $\begin{array}{l}\text { Surg + XRT } \\
\quad+\text { Embol }\end{array}$ & 4.5 & KOSCHI score & KOSCHI score $<5$ & $\begin{array}{l}\mathrm{ICH} \text { vol }>30 \mathrm{~cm}^{3} \text {, deep } \\
\text { venous drainage }\end{array}$ & $\begin{array}{l}\text { Rebleeding, SM Grade } \\
\geq \mathrm{III} \text {, associated aneu- } \\
\text { rysm, IVH, HCP, FND, } \\
\text { coma at presentation }\end{array}$ \\
\hline $\begin{array}{l}\text { Nerva et al., } \\
2016\end{array}$ & 40 & $\begin{array}{l}\text { Surg + XRT } \\
\quad+\text { Embol }\end{array}$ & 2.0 & $\begin{array}{l}\text { mRS score, obliteration } \\
\text { rate, complication rate }\end{array}$ & mRS score $>2$ & NA & NA \\
\hline
\end{tabular}

Add'I = additional; FND = focal neurological deficit; $\mathrm{HCP}$ = hydrocephalus; $\mathrm{KOSCHI}=$ King Outcome Scale for Childhood Head Injury.

* Number of pediatric patients.

$\dagger$ Values for duration of follow-up are means unless otherwise indicated.

$\ddagger$ Median.

trast-a multimodality-treated population, with an average $\mathrm{mRS}$ score of 1.35 at most recent follow-up and 21 (80.8\%) of 26 patients having an mRS score of 2 or less.

There are multiple limitations to the present study that set the stage for a prospective design. First, we limited our data collection to interviews with the patients' parents, mostly because we felt that there were strong limitations to conducting phone interviews with children. There are data to suggest that the parent proxy report differs from the patient/child report, particularly in the physical health domain..$^{19}$ Thus it would be useful in future studies to obtain information from both parents and children. This is particularly relevant in our study, where interviews with mothers were associated with a worse outcome score than interviews with fathers. The authors of the prior studies of CNS-disease HRQL summarized in Table 4 have not commented on whether there were differences between the 2 parents, and in some studies only 1 parent was routinely interviewed..$^{1,3,20}$ Rather, parent scores are averaged and occasionally compared with children values, in which case parents tend to generate lower HRQL scores than children. ${ }^{15}$ This phenomenon has been observed in other disease models as well., ${ }^{5,22}$ This is the first report to our knowledge of interparent differences, although a stronger experimental design would include collection of survey scores from both parents of each patient. Also, because the study is retrospective in nature, there is inherent bias associated with data collection. For example, a parent of a persistently neurologically disabled child interviewed 6 years after presentation may have a more negatively influenced opinion than a parent interviewed at discharge, who might still have hope for neurological recovery. Additionally, with a retrospective design, follow-up time periods vary, limiting comparisons of patient-to-patient scores (i.e., longer recovery times may produce more neurological recovery). Thus, prospective studies should aim to standardize the time points for surveying patients with bAVMs and their parents.

\section{Conclusions}

In summary, a review of the literature demonstrates that long-term clinical outcomes for pediatric bAVM patients are under-analyzed. There is a lack of consensus as to which parameters to employ; most authors use conventional metrics (i.e., mRS, GOS, PCPC, POPC) that have been validated in the adult population but not in pediatric cohorts. These metrics can serve as a surrogate for PedsQL HRQL scores. An accurate understanding of overall health-related quality of life is contingent on utilizing validated toolsets, such as the PedsQL.

\section{References}

1. Anderson V, Spencer-Smith M, Leventer R, Coleman L, Anderson P, Williams J, et al: Childhood brain insult: can age at insult help us predict outcome? Brain 132:45-56, 2009 
2. Beslow LA, Licht DJ, Smith SE, Storm PB, Heuer GG, Zimmerman RA, et al: Predictors of outcome in childhood intracerebral hemorrhage: a prospective consecutive cohort study. Stroke 41:313-318, 2010

3. Bhat SR, Goodwin TL, Burwinkle TM, Lansdale MF, Dahl GV, Huhn SL, et al: Profile of daily life in children with brain tumors: an assessment of health-related quality of life. J Clin Oncol 23:5493-5500, 2005

4. Blauwblomme T, Bourgeois M, Meyer P, Puget S, Di Rocco F, Boddaert N, et al: Long-term outcome of 106 consecutive pediatric ruptured brain arteriovenous malformations after combined treatment. Stroke 45:1664-1671, 2014

5. Britto MT, Kotagal UR, Chenier T, Tsevat J, Atherton HD, Wilmott RW: Differences between adolescents' and parents' reports of health-related quality of life in cystic fibrosis. Pediatr Pulmonol 37:165-171, 2004

6. Bull KS, Liossi C, Peacock JL, Yuen HM, Kennedy CR: Screening for cognitive deficits in 8 to 14-year old children with cerebellar tumors using self-report measures of executive and behavioral functioning and health-related quality of life. Neuro Oncol 17:1628-1636, 2015

7. Darsaut TE, Guzman R, Marcellus ML, Edwards MS, Tian L, Do HM, et al: Management of pediatric intracranial arteriovenous malformations: experience with multimodality therapy. Neurosurgery 69:540-556, 2011

8. deVeber GA, MacGregor D, Curtis R, Mayank S: Neurologic outcome in survivors of childhood arterial ischemic stroke and sinovenous thrombosis. J Child Neurol 15:316-324, 2000

9. Di Rocco C, Tamburrini G, Rollo M: Cerebral arteriovenous malformations in children. Acta Neurochir (Wien) 142:145158,2000

10. Fiser DH: Assessing the outcome of pediatric intensive care. J Pediatr 121:68-74, 1992

11. Garza-Mercado R, Cavazos E, Tamez-Montes D: Cerebral arteriovenous malformations in children and adolescents. Surg Neurol 27:131-140, 1987

12. Ghotra SK, Johnson JA, Qiu W, Newton A, Rasmussen C, Yager JY: Age at stroke onset influences the clinical outcome and health-related quality of life in pediatric ischemic stroke survivors. Dev Med Child Neurol 57:1027-1034, 2015

13. Gross BA, Storey A, Orbach DB, Scott RM, Smith ER: Microsurgical treatment of arteriovenous malformations in pediatric patients: the Boston Children's Hospital experience. J Neurosurg Pediatr 15:71-77, 2015

14. Kolb B: Synaptic plasticity and the organization of behaviour after early and late brain injury. Can J Exp Psychol 53:6276, 1999

15. Kuhlthau KA, Pulsifer MB, Yeap BY, Rivera Morales D, Delahaye J, Hill KS, et al: Prospective study of health-related quality of life for children with brain tumors treated with proton radiotherapy. J Clin Oncol 30:2079-2086, 2012

16. Majumdar M, Tan LA, Chen M: Critical assessment of the morbidity associated with ruptured cerebral arteriovenous malformations. J Neurointerv Surg 8:163-167, 2016

17. Max JE, Bruce M, Keatley E, Delis D: Pediatric stroke: plasticity, vulnerability, and age of lesion onset. J Neuropsychiatry Clin Neurosci 22:30-39, 2010

18. Nerva JD, Kim LJ, Barber J, Rockhill JK, Hallam DK, Ghodke BV, et al: Outcomes of multimodality therapy in pediatric patients with ruptured and unruptured brain arteriovenous malformations. Neurosurgery 78:695-707, 2016

19. Ramachandra P, Palazzi KL, Skalsky AJ, Marietti S, Chiang G: Shunted hydrocephalus has a significant impact on quality of life in children with spina bifida. PM R 5:825-831, 2013
20. Rivara FP, Vavilala MS, Durbin D, Temkin N, Wang J, O'Connor SS, et al: Persistence of disability 24 to 36 months after pediatric traumatic brain injury: a cohort study. J Neurotrauma 29:2499-2504, 2012

21. Sanchez-Mejia RO, Chennupati SK, Gupta N, Fullerton H, Young WL, Lawton MT: Superior outcomes in children compared with adults after microsurgical resection of brain arteriovenous malformations. J Neurosurg 105 (2 Suppl):82-87, 2006

22. Sheffler LC, Hanley C, Bagley A, Molitor F, James MA: Comparison of self-reports and parent proxy-reports of function and quality of life of children with below-the-elbow deficiency. J Bone Joint Surg Am 91:2852-2859, 2009

23. Singhal A, Adirim T, Cochrane D, Steinbok P: Pediatric patients with poor neurological status and arteriovenous malformation hemorrhage: an outcome analysis. J Neurosurg Pediatr 7:462-467, 2011

24. Swanson JO, Vavilala MS, Wang J, Pruthi S, Fink J, Jaffe $\mathrm{KM}$, et al: Association of initial CT findings with quality-oflife outcomes for traumatic brain injury in children. Pediatr Radiol 42:974-981, 2012

25. Varni JW, Burwinkle TM, Seid M, Skarr D: The PedsQL 4.0 as a pediatric population health measure: feasibility, reliability, and validity. Ambul Pediatr 3:329-341, 2003

26. Varni JW, Limbers CA: The pediatric quality of life inventory: measuring pediatric health-related quality of life from the perspective of children and their parents. Pediatr Clin North Am 56:843-863, 2009

27. Varni JW, Seid M, Kurtin PS: PedsQL 4.0: Reliability and validity of the Pediatric Quality of Life Inventory version 4.0 generic core scales in healthy and patient populations. Med Care 39:800-812, 2001

28. Wong ST, Fong D: Ruptured brain arteriovenous malformations in children: correlation of clinical outcome with admission parameters. Pediatr Neurosurg 46:417-426, 2010

29. World Health Organization: International Classification of Functioning, Disability and Health. Geneva: World Health Organization, 2001

\section{Disclosures}

The authors report no conflict of interest concerning the materials or methods used in this study or the findings specified in this paper.

\section{Author Contributions}

Conception and design: Sekhar, Nerva, Rockhill, Kim. Acquisition of data: Abecassis, Nerva, Rockhill, Kim. Analysis and interpretation of data: Sekhar, Abecassis, Barber, Ellenbogen, Kim. Drafting the article: Sekhar, Abecassis, Nerva. Critically revising the article: Sekhar, Abecassis, Nerva, Ellenbogen. Reviewed submitted version of manuscript: Sekhar, Abecassis, Nerva, Barber, Ellenbogen, Kim. Approved the final version of the manuscript on behalf of all authors: Sekhar. Statistical analysis: Abecassis, Barber. Administrative/technical/material support: Ellenbogen. Study supervision: Sekhar, Nerva, Ellenbogen.

\section{Correspondence}

Laligam N. Sekhar, Department of Neurological Surgery, University of Washington, Harborview Medical Center, 325 9th Ave., Box 359924, Seattle, WA 98104. email: lsekhar@u.washington.edu. 